\title{
An Evaluation of Optical Absorbance Kinetics for the Detection of Micro-Porosity in Molecularly Doped Polymer Thin-Films
}

\author{
David Mitchell Goldie *, Orlaith Skelton and Thomas James Bailey
}

School of Science and Engineering, University of Dundee, Dundee DD1 4HN, UK; o.skelton@dundee.ac.uk (O.S.); t.j.bailey@dundee.ac.uk (T.J.B.)

* Correspondence: d.m.goldie@dundee.ac.uk

\begin{abstract}
The use of optical absorbance kinetics to identify micro-porous regions in doped polymer films is evaluated. Data are presented for a series of hydrazone doped polymer films which are found to optically bleach upon exposure to an ultra violet (UV) radiation source. The UV absorbance kinetics are found to exhibit distinctive characteristics for the various polymers studied, with changes in film absorbance occurring either in a fast $\left(<10^{3} \mathrm{~s}\right)$ or slow $\left(>10^{4} \mathrm{~s}\right)$ timescale. An interpretation of these distinctive timescales based upon a cellular-automata model of the absorbance kinetics suggests that the underlying photo-oxidation of the hydrazone is highly sensitive to underlying micro-porosity in the films which controls the necessary supply of absorbed oxygen for photo-cyclic reaction.
\end{abstract}

Keywords: molecularly doped polymer; optical absorbance kinetics; micro-porosity

\section{Introduction}

Thin-film organic materials have become increasingly important as the active elec-

Citation: Goldie, D.M.; Skelton, $\mathrm{O}$ Bailey, T.J. An Evaluation of Optical Absorbance Kinetics for the Detection of Micro-Porosity in Molecularly Doped Polymer Thin-Films. Solids 2021, 2, 155-164. https://doi.org/ $10.3390 /$ solids 2020010

Academic Editor: Antonio Polimeni

Received: 1 February 2021

Accepted: 29 March 2021

Published: 1 April 2021

Publisher's Note: MDPI stays neutral with regard to jurisdictional claims in published maps and institutional affiliations.

tronic medium in a wide range of large-area imaging and display devices. An important class of such organic materials are molecularly doped polymers (MDPs) which comprise an electronically inert polymer binder into which an electrically active dopant molecule is dispersed. Careful chemical selection of the polymer-dopant combination, and optimisation of the dopant concentration, permits the benchmark mobility-lifetime $(\mu \tau)$ product to be sensitively controlled for the majority electronic carrier according to the target application. The $\mu \tau$ product has historically been recognized as a significant controlling parameter for the achievable efficiency of thin-film inorganic solar cells [1,2], and more recently in the development of optimized thin-film organic solar cells [3] and large-volume crystalline detectors for ionizing radiation [4]. The long-term stability of such $\mu \tau$ magnitudes in MDPs may be seriously compromised, however, via unwanted internal chemical reactions. Such reactions are generally induced by internal photo-excitation processes and are significantly more damaging for the dopant molecules which are subsequently rendered to become electronically inactive.

Mitigation of photo-degradation processes in MDP thin-films consequently demands that a comprehensive understanding of the underlying processes and parameters that are dominant in driving these internal processes are fully identified and numerated. Previous work in this area has focused upon a specific photo-cyclic degradation process that is known to occur for a small hydrazone molecule p-diethylaminobenzaldehyde $-1,1^{\prime}$ diphenylhydrazone (DEH) in MDP thin films [5-8]. The photo-cyclic conversion of DEH to the electronically inactive indazole (IND) product requires that the DEH molecule be first excited into a (reactive) state by appropriate absorption of UV radiation, and that excited DEH molecules have a supply of absorbed oxygen to then complete the reactive transition to IND. Modelling of the conversion dynamics of DEH to IND has previously been undertaken using rate-equation [7] and cellular-automata (CA) approaches [8]. From these studies it was found that the conversion dynamics of the hydrazone molecular dopant to 
indazole photo-product $(P)$ could be analytically described as a function of photo-exposure time $(t)$ by a stretched-exponential function such that:

$$
P(t)=\Gamma\left[1-e^{-\left(\frac{t}{\tau}\right)^{\gamma}}\right]
$$

In Equation (1) the fitting parameters $\Gamma$ and $\gamma$ are expected to be close to unity [8] provided the concentration of dopant $\left(\mathrm{c}_{\mathrm{M}}\right)$ is kept sufficiently low $(<20 \%$ by weight relative to the polymer). The other parameter $\tau$ in Equation (1) represents a characteristic time constant given by $\tau=\mathrm{c}_{\mathrm{M}} / \mathrm{c}_{\mathrm{O}} \beta$ where $\mathrm{c}_{\mathrm{O}}$ represents the concentration of soluble oxygen, and $\beta$ gives the molecular relaxation rate of reactive DEH back to the (un-reactive) ground state. It is noted that short degradation time's $\tau$ may therefore be facilitated by high levels of soluble oxygen $c_{O}$ within the MDP film. Polymer morphologies which facilitate high soluble oxygen levels, such as those that contain micro-porous channels, should accordingly be avoided when identifying potential dopant-polymer pairs for MDP applications.

A consequence of the dynamical increase in IND photo-product with exposure time according to Equation (1) is that the MDP films are expected to photo-bleach if, relative to the absorption strength of the DEH molecule, the IND molecule is relatively transparent at the selected UV excitation wavelength [6]. A simple experimental methodology to investigate the conversion dynamics is therefore presented by selecting an appropriate UV exposure wavelength which may serve the dual function of initiating the photo-excitation process and providing a reference transmission signal through the film to determine the optical absorbance. As noted from previous cellular-automata simulations of the absorbance dynamics [8] the overall change in absorbance will then be determined by the changing proportion of DEH to IND as photo-exposure progress, and will be sensitive to the relative magnitude of the DEH and IND molecular absorption coefficients for the film thickness used. The proposed absorption approach may consequently offer a simple, affordable approach to investigate the porosity of thin polymer films which may be inaccessible using traditional bulk material methods [9-11] or which require the use of freestanding films [10-12]. Experimental absorption data to test these model predictions have now been acquired and are presented in the present work. In particular the potential use of the technique to evaluate and identify thin-film polymers which may contain micro-porous regions, and would thus be unsuitable for MDP consideration, is evaluated.

\section{Materials and Methods}

A series of DEH doped MDP films were deposited onto glass substrates (area $=6.25 \mathrm{~cm}^{2}$ ) for the optical absorbance experiments. The polymers used for these MDPs were a polyester (PE; Vitel 2000), a polycarbonate (PC; Lexan 161) and a polystyrene (PS). The polymer materials and the DEH were sourced from a commercial supplier and used as received without further purification. Solutions of these polymers were prepared using analyticalgrade dichloromethane into which were co-dissolved specified percentage amounts by weight of the DEH to polymer. Thin-films of these co-solutions were cast onto the glass substrates using an industrial spin-coater unit. The achieved film thicknesses (L), and associated surface roughness of the films $(\Delta \mathrm{L})$, were determined using a surface profilimeter unit following a $24 \mathrm{~h}$ drying period under ambient conditions. To minimise unintentional exposure of the MDP films during preparation these procedures were conducted in a UV-safe photolithographic clean-room environment. A summary of the prepared MDP films is given in Table 1 . The column entries for $\varphi_{f}, \tau_{f}$ and $\tau_{s}$ in Table 1 represent parameters that have been determined from analysis of the absorbance kinetics for these films and are defined in the results section.

A schematic diagram that shows how UV absorbance measurements were conducted using these films is given in Figure 1. A suitable commercial UV LED was identified to simultaneously perform the exposure of the deposited films and allow the optical absorbance to be monitored. The selected LED had a peak wavelength of $385 \mathrm{~nm}$ and a spectral width of $\pm 5 \mathrm{~nm}$. This wavelength provided a good match to promote electrons from the DEH 
highest occupied molecular orbital (HOMO) to the lowest occupied molecular orbital (LUMO) [13]. Only the DEH dopant was found to be strongly absorbing at this wavelength as desired. All of the constituent MDP polymers, the IND photo-degradation product, and the supportive glass substrate were determined to be transparent at this incident wavelength. The LED was powered to supply an incident UV flux at the surface $(x=0)$ of the MDP films corresponding to $\mathrm{UV}_{0} \sim 10^{16}$ photons $\mathrm{cm}^{-2} \mathrm{~s}^{-1}$ in Figure 1. The incident UV light was confined within a circular region of $3 \mathrm{~mm}$ diameter using an optical mask applied to the surface of the film. This permitted absorbance measurements to be repeated at multiple locations across the available film area and the reproducibility of acquired data to be assessed.

Table 1. Details of the MDP samples used to undertake the spectral and absorbance measurements.

\begin{tabular}{ccccccc}
\hline Polymer & $\mathbf{c}_{\mathbf{M}}(\mathbf{\%})$ & $\mathbf{L}(\boldsymbol{\mu \mathbf { m }})$ & $\boldsymbol{\Delta} \mathbf{L}(\boldsymbol{\mu m})$ & $\boldsymbol{\varphi}_{f}$ & $\boldsymbol{\tau}_{f}(\mathbf{s})$ & $\tau_{\boldsymbol{s}}(\mathbf{s})$ \\
\hline PE & 10 & 2.5 & 0.3 & 0 & & $2 \times 10^{4}$ \\
PE & 10 & 1.0 & 0.2 & 0 & & $9 \times 10^{3}$ \\
PE & 20 & 2.3 & 0.4 & 0 & & $5 \times 10^{4}$ \\
PE & 20 & 1.1 & 0.2 & 0 & & $2 \times 10^{4}$ \\
PE & 30 & 1.4 & 0.2 & & & \\
PE & 40 & 1.8 & 0.3 & & & \\
PE & 50 & 2.2 & 0.6 & & & \\
PC & 10 & 1.1 & 0.1 & 0.73 & $3 \times 10^{2}$ & $7 \times 10^{4}$ \\
PC & 20 & 1.3 & 0.3 & 0.80 & $6 \times 10^{2}$ & $5 \times 10^{4}$ \\
PS & 10 & 1.1 & 0.3 & 0.80 & $3 \times 10^{2}$ & $7 \times 10^{4}$ \\
PS & 20 & 0.9 & 0.2 & 0.81 & $7 \times 10^{2}$ & $5 \times 10^{4}$ \\
\hline
\end{tabular}

G MDP

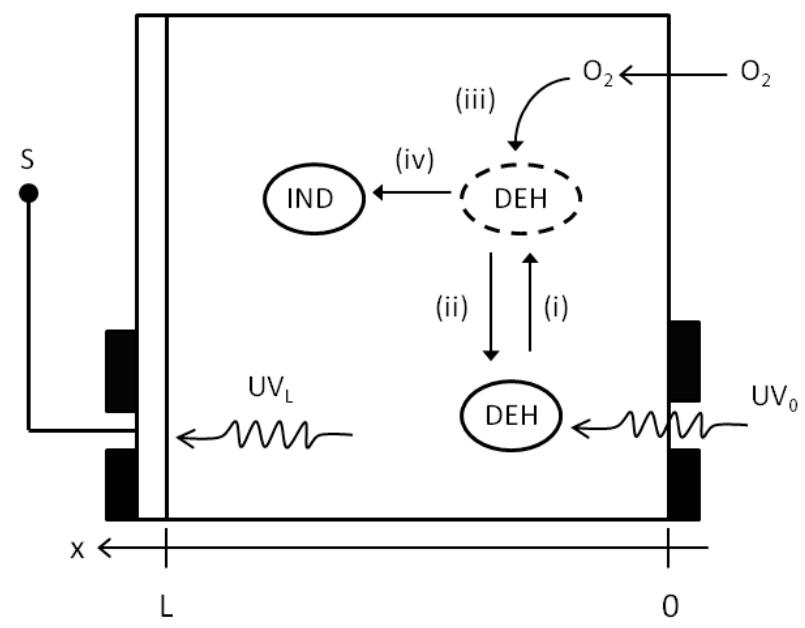

Figure 1. Experimental configuration used to perform UV absorption measurements on MDP films of thickness $\mathrm{L}$. $\mathrm{UV}_{0}$ and $\mathrm{UV}_{\mathrm{L}}$ are, respectively, the incident $\mathrm{UV}$ intensity at the film surface and transmitted through the film thickness. The internal photo-cyclic oxidation processes for $\mathrm{DEH}$ are indicated according to processes (i)-(iv) described in the discussion section. $G=$ glass substrate, $\mathrm{S}=$ spectrometer.

A UV-IR spectrometer unit (detection range $300 \mathrm{~nm}$ to $1100 \mathrm{~nm}$, resolution $\pm 0.4 \mathrm{~nm}$ ) was used to undertake both spectral scanning of the transmitted light through the MDP films and absorbance measurements in a kinetics mode. Spectral scans were recorded in a restricted wavelength range from $300 \mathrm{~nm}$ to $475 \mathrm{~nm}$ for MDP films which were initially un-exposed, and then re-taken following full UV exposure conditions. In the spectrometer kinetics mode an integrated signal $\left(\mathrm{UV}_{\mathrm{L}}\right.$ at $\mathrm{x}=\mathrm{L}$ in Figure 1) between $380 \mathrm{~nm}$ and $390 \mathrm{~nm}$ was used to monitor the relative change in the absorbance as the films were continuously 
exposed over a period up to a maximum of $4 \times 10^{5} \mathrm{~s}$. Coupling of the transmitted light to the spectrometer unit was achieved via a fibre-optic cable under all operational modes. The use of the fibre-optic cable did not permit an integrating sphere to be incorporated into the experimental setup and the film absorbance (A) was consequently evaluated by the amount of transmitted light according to $A=-\log \left[\mathrm{UV}_{\mathrm{L}} / \mathrm{UV}_{0}\right]$. All spectral and kinetic mode measurements were conducted under ambient room-temperature $(295 \mathrm{~K})$ conditions with the MDP specimens and attached LED located in a light-secure container to ensure that film exposure only occurred via the experimental UV source.

\section{Results}

The results obtained by performing spectral scans on a series of unexposed PE MDP films are shown in Figure 2. Data are included for DEH doping concentrations that range from $c_{M}=10 \%$ to $c_{M}=50 \%$. The spectral output from the UV LED is also included for reference. Relative to the UV reference the spectral responses recorded for the MDP films are observed to be red shifted to longer wavelengths. Similar spectral red shifting was detected in the recorded spectra for unexposed PC and PS MDP films.

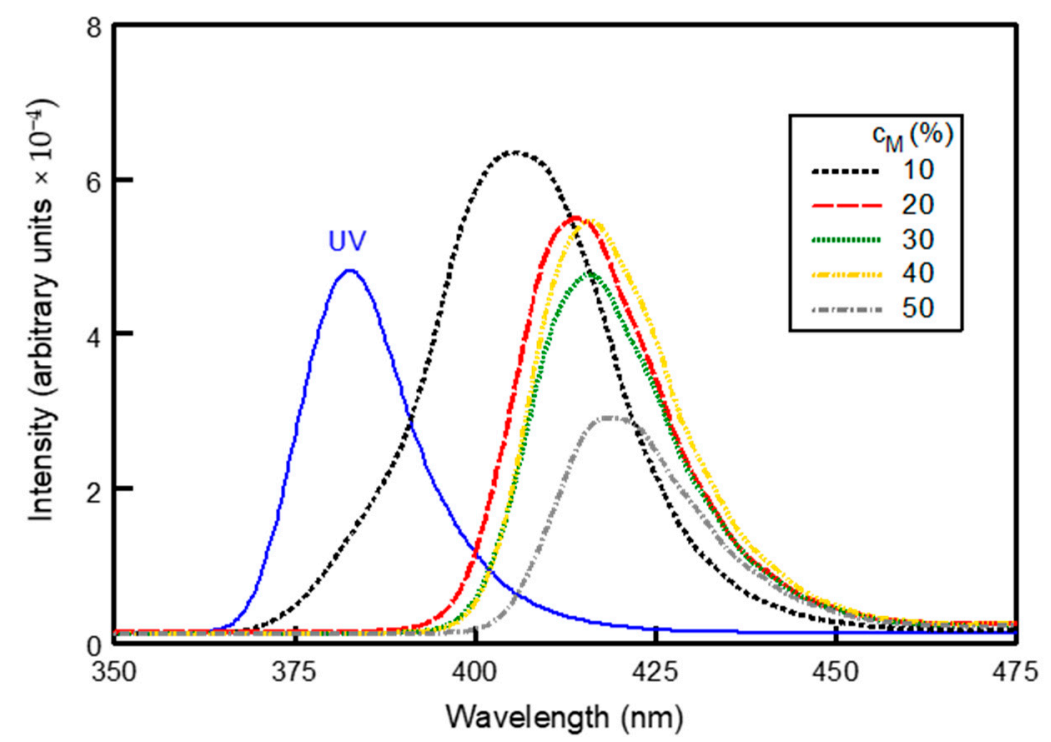

Figure 2. UV excited spectra for a series of unexposed DEH doped PE films at the \% doping concentrations as indicated. The UV LED emission is included for reference.

Spectral scans were repeated on the MDPs following a full exposure period $\left(4 \times 10^{5} \mathrm{~s}\right)$ to the UV LED. The scans obtained for the PE MDPs are given in Figure 3 for the $\mathrm{c}_{\mathrm{M}}=10 \%$ and $c_{M}=20 \%$ films. In this plot the UV reference has again been included together with the original unexposed scans from Figure 2 at these doping concentrations. Compared to the unexposed scans, the scans obtained from the fully exposed films no longer exhibit the original red shift but are now observed to be in the same range of the UV reference.

The results of an early optical absorbance study that was undertaken to evaluate how reproducible the dynamical data recorded across several regions of an MDP film would be are presented in Figure 4. These data were recorded using the spectrometer in the kinetics mode for a $2.5 \mu \mathrm{m}$ thick PE MDP film $\left(\mathrm{c}_{\mathrm{M}}=10 \%\right)$ by applying the optical mask at various randomly selected regions. The data show the change in (UV) absorbance relative to the reference absorbance at time $t=0$ in the un-exposed state. The co-incidence of the data curves suggests that the LED output flux remains stable throughout the long dynamical recording period and that the film composition and thickness is uniform across the entire film area. 


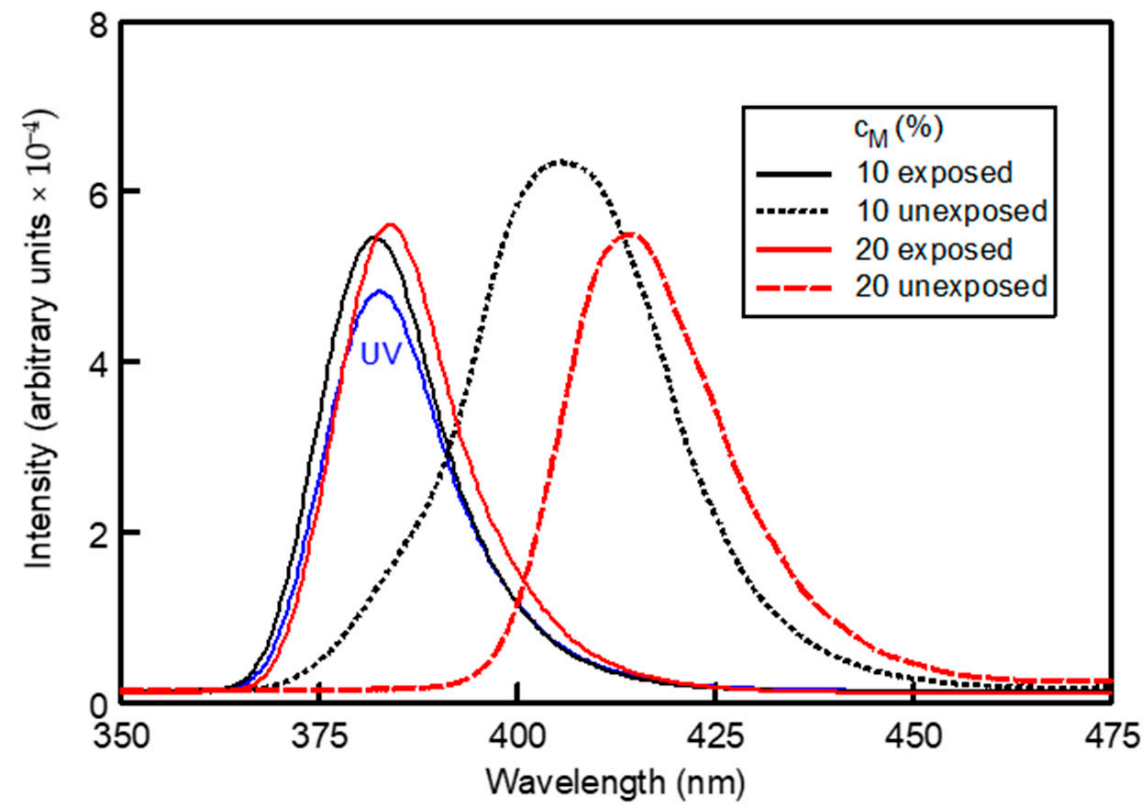

Figure 3. UV excited spectra for $10 \%$ and $20 \%$ doped DEH PE films in the unexposed and fully exposed states. The UV LED emission is included for reference.

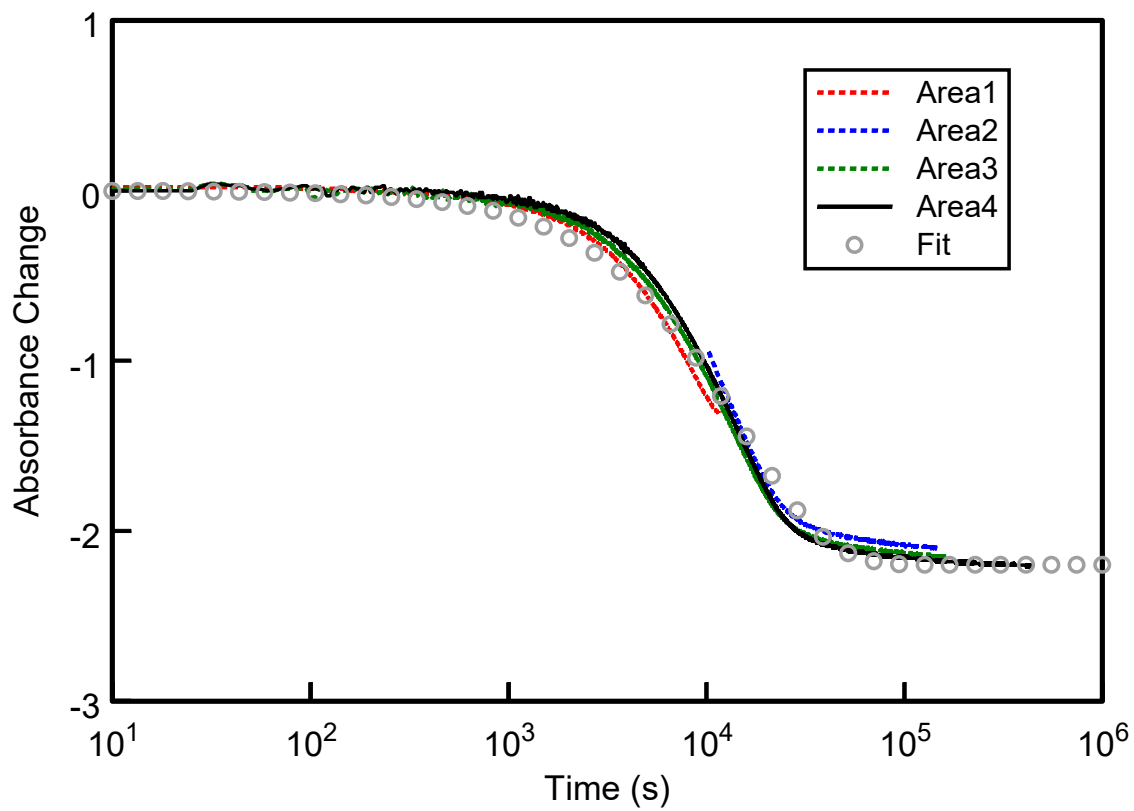

Figure 4. Absorbance kinetics recorded for the $2.5 \mu \mathrm{m}$ thick PE sample in Table 1 at different regions across the film surface. The open circles show the optimum fit to the composite data using Equation (3) and the parameters for the $P(t)$ function in Equation (2) as described in the main text.

Further absorbance data were recorded for the other MDP films listed in Table 1. Examples of the dynamical responses obtained for the $c_{M}=20 \%$ films are shown in Figure 5 . These data sets (shown as the solid lines) were all acquired using the standard UV flux from the LED source applied at a randomly selected location on the film surface. The symbols on this figure have been generated using an analytical expression which is described in the discussion that follows. 


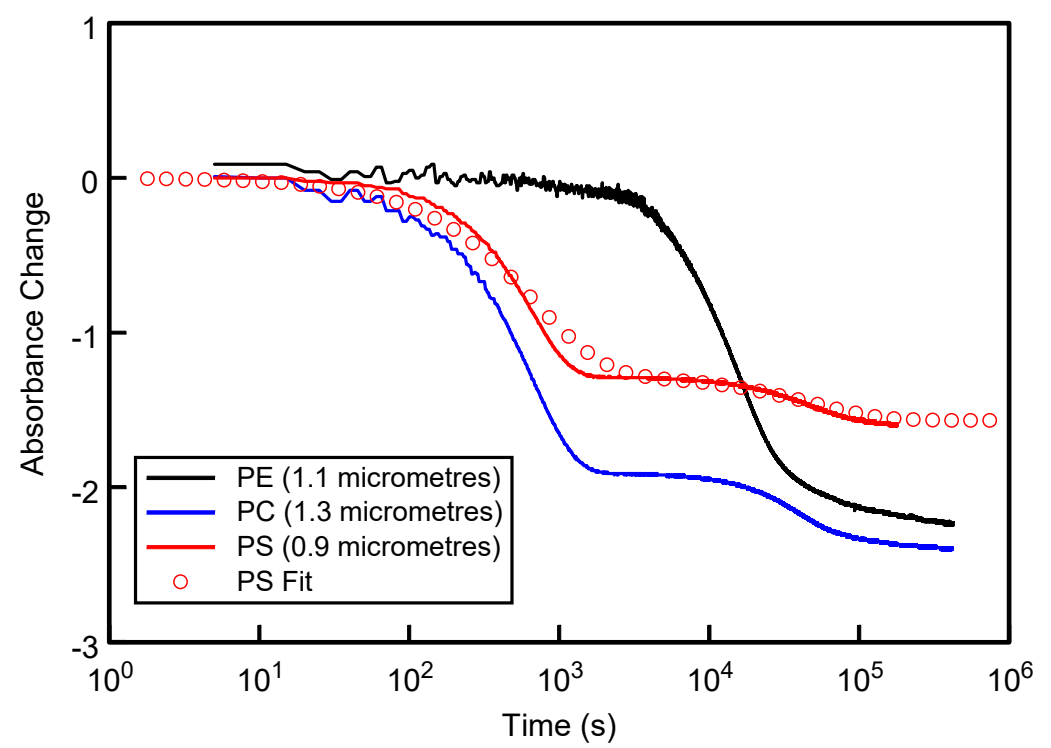

Figure 5. Absorbance kinetics in a series of $20 \%$ DEH doped polymer films. The open circles show the optimum fit of Equation (3) to the PS data using the parameters given in Table 1. Note that in applying Equation (3) the modified expression $P^{\prime}(t)$ from Equation (4) has been used as outlined in the main text.

\section{Discussion}

The significant red-shift observed in Figure 2 for the unexposed PE MDP spectra is consistent with the DEH excitation (i) and relaxation (ii) processes depicted in Figure 1. In the unexposed state these MDP films are initially strongly absorbing at the UV wavelength and virtually none of this incident radiation is therefore transmitted to the spectrometer. Sufficiently short exposure times of typically $<10$ s are adequate to permit the spectra to be experimentally recorded, yet ensure that the majority of the excited DEH molecules subsequently relax to their original ground state via process (ii) in preference to reacting with soluble oxygen to form IND (processes (iii) and (iv) in Figure 1). The relaxation process (ii) will consequently emit a photon but this is expected to be at a lower energy as the excited electrons thermalise to the lowest energy states within the DEH LUMO band. For MDP materials the LUMO and HOMO Gaussian bandwidths are expected to increase with the dopant concentration due to random dipole disorder [8,14]. For the DEH molecule which possesses a dipole moment $~ 2.1$ debye this results in a bandwidth of around $0.11 \mathrm{eV}$ for $\mathrm{c}_{\mathrm{M}}=50 \%$ [14]. For energetically symmetric LUMO and HOMO bands the net reduction in emitted photon energy during process (ii) is therefore expected to be around $0.22 \mathrm{eV}$ with an associated red shift of about $30 \mathrm{~nm}$ relative to the incident UV peak. This is quantitatively consistent with the data in Figure 2 where the peak for the $\mathrm{c}_{\mathrm{M}}=50 \%$ occurs at about $419 \mathrm{~nm}$. The spectral red shift is furthermore observed to become progressively smaller as $\mathrm{c}_{\mathrm{M}}$ is reduced which is expected due to the associated dipole disorder narrowing of the HOMO and LUMO bandwidths.

For fully exposed films the spectral response is anticipated to be significantly different due to the conversion of DEH to IND. This is confirmed for the lightly doped PE MDP specimens in Figure 3 where spectra for the fully exposed and unexposed films are compared. In contrast to the red-shifted spectra recorded at the start of the exposure period, the fully exposed films are now observed to have a spectral signature that matches the incident UV profile. This confirms that the films are now entirely transparent to the incident UV radiation and have therefore become UV bleached due to the replacement of strongly absorbing DEH molecules with the non-absorbing IND photo product.

The spectral phenomena observed in Figures 2 and 3 suggest that it should therefore be possible to dynamically monitor the DEH to IND photo-conversion process by either recording the intensity of the emitted red-shifted radiation or the amount of transmitted UV 
LED light. Consideration of the spectrometer's signal gain requirements when operated in a kinetic acquisition mode indicated that monitoring of the transmitted UV signal was more reliable, however, and permitted a greater range of film absorbance to be monitored across the maximum exposure period. The absorbance data presented in Figures 4 and 5 have consequently been determined using this reference UV signal approach.

As already noted for the Figure 4 data, the recorded change in absorbance was found to be highly reproducible across randomly selected regions of the film. The overall reduction in absorbance for this specimen corresponds to an increase by a factor of around 160 in received UV light at the spectrometer once exposure is completed. Such a large increase in signal proved challenging to process using the spectrometer's fixed-gain setting in the kinetics mode as the gain required at the start of the exposure period to reliably establish a reference baseline would sometimes result in detector saturation before full-exposure had been completed. To experimentally mitigate this issue specimens were latterly prepared with a reduced target thickness of around $1 \mu \mathrm{m}$ so that the starting reference signals could be obtained using a minimal spectrometer gain. It is consequently suggested that a bespoke experimental setup that comprises a UV-sensitive photodiode operating with a transimpedance amplifier would provide an optimised setup to record this type of dynamical absorbance data. Evaluation of such a proto-type system using a micro-controller and digital potentiometers in the feedback loop to optimise the signal gain has provided similar absorbance results to those obtained from the spectrometer unit. The overall cost of such a bespoke system is presently estimated to be around $5-10 \%$ of the spectrometer's listed purchase price.

Whilst the absolute absorbance change over the exposure period presents many interesting experimental challenges, the associated timescale and temporal signature for the change contains potentially important information about the underlying polymer morphology. The overall change in the film absorbance $(\delta A)$ with time may be functionally expressed as $\delta A(t)=-\log \left[\mathrm{I}(t) / \mathrm{I}_{0}\right]$ where $\mathrm{I}_{0}$ and $\mathrm{I}(t)$ are, respectively, the intensities of $\mathrm{UV}$ light received at the spectrometer at the start $(t=0)$ of the exposure period and after an exposure period $t$ has elapsed. Assuming a simple de Beer's law dependence for UV absorption through the film thickness permits $\delta A(t)$ to then be expressed in terms of the film absorption coefficient $(\alpha)$ as:

$$
\delta A(t)=-\log \left[\exp \left(\left(\alpha_{0}-\alpha(t)\right) L\right)\right]
$$

In Equation (2) $L$ is again the film thickness, and $\alpha_{0}$ and $\alpha(t)$, respectively, represent the corresponding film absorption coefficients at the start of the exposure period, and following an interval $t$ of $U V$ exposure. The overall absorption coefficient for the film is entirely controlled by specific UV absorption events by DEH and IND molecules, however, and may consequently be expressed in terms of the associated absorption coefficients for these respective molecular species $\left(\alpha_{D E H}\right.$ and $\left.\alpha_{I N D}\right)$. If these molecular absorption events occur independently then the overall film absorption coefficient may be estimated from the weighted proportion of DEH to IND that exists in the film at any time during exposure. From Equation (1) it follows that $\alpha(t)=[1-P(t)] \alpha_{D E H}+P(t) \alpha_{I N D}$ and using this in Equation (2) finally yields the following expression for the absorbance dynamics:

$$
\delta A(t)=-\log \left[\exp \left(P(t)\left(\alpha_{D E H}-\alpha_{I N D}\right) L\right)\right]
$$

It is noted from Equation (3) that since $P(t)$ increases with exposure time, then the film absorbance is expected to decrease (UV bleaching) provided $\alpha_{D E H}>\alpha_{I N D}$. The maximum relative change in absorbance, which is acquired after full exposure when $P(t) \rightarrow \Gamma$, will then be dictated by the film thickness L. The selection of an appropriate film thickness is therefore expected to be key in achieving an appropriate response range for $\delta A$ but whilst it is tempting to use thicker films this may have experimental consequences as already noted in the preceding discussion concerning the spectrometer gain. 
The ability of Equation (3) to fit experimental data is demonstrated in Figure 4 for the $2.5 \mu \mathrm{m}$ thick polyester film where $\mathrm{c}_{\mathrm{M}}=10 \%$. The optimum fit as shown by the open symbols returns values of $\Gamma=1.0$ and $\gamma=0.9$ in accordance with the low-doping expectations previously noted for the $P(t)$ function [8]. The characteristic time constant is then $\tau=2 \times 10^{4} \mathrm{~s}$, whilst the overall change in the absorbance for the nominal film thickness requires that $\alpha_{I N D} / \alpha_{D E H}=0.1$ using a value of $\alpha_{D E H}=3 \times 10^{4} \mathrm{~cm}^{-1}$ determined from optical transmission measurements. Whilst there is a reasonable level of confidence in the fitted parameters $(\Gamma, \gamma$ and $\tau)$ that control the temporal shape of the absorbance change, it is evident that uncertainties associated with the film thickness ( $\triangle \mathrm{L}$ in Table 1$)$ and the appropriate magnitude for $\alpha_{D E H}$ may result in unacceptably large values for $\alpha_{I N D}$ being returned to account for the net change in absorbance. A constraint that $\alpha_{I N D} / \alpha_{D E H}$ should remain sufficiently low $(\leq 0.1)$ during the fitting procedure was consequently applied to comply with the previously noted observation [6] that the IND molecule is optically "transparent" at the incident UV wavelength. This additional constraint on the fitting procedure was not, however, found to significantly affect the emergent $P(t)$ parameters $(\Gamma, \gamma$ and $\tau)$. The further application of Equation (3) to fit experimental data in a more heavily doped polyester film $\left(c_{M}=20 \%\right)$ is shown if Figure 5 where comparable parameters are found for $\Gamma$ and $\gamma$ and a similar value of $\tau=2 \times 10^{4} \mathrm{~s}$ is extracted. The similarity of these time constants does not initially appear to be consistent with the CA expression $\tau=c_{M} / c_{O} \beta$ where it is anticipated that under identical experimental conditions the required photo-degradation time will scale linearly with the amount of DEH dopant provided $\mathrm{c}_{\mathrm{M}}<40 \%$ [8]. As the film thicknesses are significantly different for the PE data presented in Figures 4 and 5, the simple $\times 2$ scaling factor predicted from $c_{M}$ considerations alone is therefore not observed (there is simply more DEH present in the thicker $c_{M}=10 \%$ film which requires a longer time to degrade under equivalent UV exposure conditions). The expected time constant scaling with $\mathrm{c}_{\mathrm{M}}$ is, however, confirmed for the PE films in Table 1 which have comparable thicknesses. Here, the time constant values for the $c_{M}=10 \%$ and $c_{M}=20 \%$ PE films have been entered for convenience under the column headed $\tau_{s}$ for reasons that are described below.

It is evident that for the other data presented in Figure 5 for the polystyrene and polycarbonate films Equation (3) will no longer provide an appropriate description of the absorbance dynamics as these films appear to exhibit a 2-stage reduction in $\delta A$ that occur at distinctive times. Compared to the single-stage polyester response, the initial fall of $\delta A$ for these films occur at significantly shorter times which suggests that fast conversion to IND occurs at a proportion of the DEH sites (stage 1) before a slower conversion of the remaining DEH sites is detected at later times (stage 2). An obvious modification to Equation (3) to permit this 2-stage process to be analytically modelled is to simply replace the $P(t)$ element from Equation (1) with an equivalent weighted expression for the fast and slow DEH components. Assuming that the degradation processes within these DEH components are independent the modified replacement for $P(t)$ would then simply be:

$$
P^{\prime}(t)=\Gamma \phi_{f}\left[1-e^{-\left(\frac{t}{\tau_{f}}\right)^{\gamma}}\right]+\left(1-\phi_{f}\right)\left[1-e^{-\left(\frac{t}{\tau_{s}}\right)^{\gamma}}\right]
$$

In Equation (4) the parameter $\varphi_{f}$ represents the proportion of DEH sites which may undergo fast degradation, and $\tau_{f}$ and $\tau_{s}$ denote the associated time constants for the fast and slow reaction sites, respectively. The ability of Equation (4) to fit the $c_{M}=20 \%$ polystyrene data is illustrated in Figure 5 where the proportion of DEH sites that undergo fast degradation is found to be around $80 \%$. Similar fitting procedures applied to the $\mathrm{c}_{\mathrm{M}}=10 \%$ polystyrene film, and equivalently doped polycarbonate specimens, return broadly similar proportions of fast to slow DEH sites as evidenced in Table 1. The associated time constants for the fast reaction sites is furthermore found to be about two orders of magnitude lower than for the slow sites for both of these polymer binders. The single time constant extracted for the polyester films falls between these magnitudes although closer to the slow values and these parameters have consequently been entered under the $\tau_{s}$ column in Table 1 for convenience with $\varphi_{f}$ consequently set to zero for this particular binder. 
It is interesting to consider the physical origin of the emergence of the significantly faster degradation process in the polystyrene and polycarbonate films which is absent in the polyester samples. As noted in the introduction, the signature time-constant $\tau=\mathrm{c}_{\mathrm{M}} / \mathrm{c}_{\mathrm{O}} \beta$ so that for equivalently doped films the shorter time-constant implies that either the concentration $\mathrm{c}_{\mathrm{O}}$ of soluble oxygen is enhanced at a fraction $\left(\varphi_{f}\right)$ of the $\mathrm{DEH}$ sites and/or the relaxation rates $\beta$ for this subset of excited DEH sites is increased [8]. Similar spectral responses to those presented for the polyester films in Figures 2 and 3 are also found for the polystyrene and polycarbonate samples, however, which suggests that the relaxation of excited DEH sites is principally controlled by direct transitions between the molecular HOMO and LUMO states. The influence of the polymer in the relaxation process is therefore likely to be minimal (other than to permit limited thermalisation within the HOMO) and the $\beta$ parameter is consequently likely to be a specific characteristic of the DEH molecule and polymer independent. Any influence of the polymer binder upon $\tau$ is thus inferred to arise mainly through the available concentration of soluble oxygen to complete the photo-cyclic conversion to IND [5].

The presence of two distinctive degradation stages in the polystyrene and polycarbonate samples would then appear to indicate that the concentration of soluble oxygen throughout the polymer is non-uniform for these samples with regions of higher $\mathrm{c}_{\mathrm{O}}$ resulting in the faster degradation process. Film morphology properties that may explain the presence of locally enhanced oxygen levels include surface topography characteristics (roughness and associated porosity) [15,16], and the presence of micro-pores within the bulk of the polymer binder [9,17]. However, the relatively large value of 0.8 deduced for the $\varphi_{f}$ parameter is inconsistent with enhanced degradation being entirely confined to a small fraction of the film thickness in the vicinity of the film surface (assuming uniform dispersal of the DEH dopant throughout the film bulk). It is furthermore noted that, for the thinner $\sim 1 \mu \mathrm{m}$ films in Table 1 , the film roughness $\Delta \mathrm{L}$ is around $20 \%$ of the film thickness for all of the polymer binders. A more likely explanation for the PS and PC behaviour is that these particular polymers contain a significant network of micro-pores throughout the film bulk that are able to maintain an enhanced concentration of oxygen across the majority $(\sim 80 \%)$ of the DEH sites. A minority ( 20\%) of DEH sites in these films are presumably more isolated from the micro-pores and thus degrade at a significantly slower rate. The PE samples must by contrast possess a more uniform morphology throughout the entire film bulk which yields the single degradation rate response. The $\tau$ magnitude found for the PE films may accordingly indicate that the underlying bulk morphology for this binder is entirely devoid of micro-pores.

Whilst the preceding arguments offer some insight into how the observed degradation dynamics may be controlled via the various MDP binder morphologies it is still important to confirm that these processes proceed independently and that Equation (4) may therefore be applied with confidence. Further cellular-automata modelling of the degradation dynamics has accordingly been undertaken in which the original CA rule-set [8] was modified to now permit a pre-defined fraction $\left(\varphi_{f}\right)$ of excited DEH cells to convert to IND cells with an enhanced probability. Such CA cells therefore constitute fast reaction sites within the MDP bulk that have an enhanced oxygen concentration supplied by micro-pores. It is found that the output generated from this modified CA approach may indeed be functionally described by Equation (4) and that the parameters returned $\left(\varphi_{f}, \tau_{f}\right.$ and $\left.\tau_{s}\right)$ are consistent with those employed by the CA rule-set $\left(\varphi_{f}, \mathrm{c}_{\mathrm{O}}\right.$ and $\left.\beta\right)$.

\section{Conclusions}

The dynamics of optical absorbance have been successfully recorded for a series of DEH MDPs and are found to be consistent with the functional predictions of previous CA modelling. The extraction of reliable polymer parameters via fitting of absorbance kinetic data is found to be sensitive, however, to accurate knowledge of the film thickness and absorption coefficient for the selected dopant molecule. Some of the polymer binders used in the present MDP films contain distinguishable fast and slow reactive DEH sites 
which may indicative the presence of micro-pores in the underlying polymer morphology. The experimental approach described may consequently offer a simple, cost-effective approach to investigate the porosity of thin polymer films which are coated onto supportive substrates and thereby also offer more convenient handling than methods which necessitate the use of free-standing films.

Author Contributions: Conceptualization, D.M.G.; Data curation, O.S.; Investigation, D.M.G. and O.S.; Methodology, O.S.; Resources, D.M.G.; Software, T.J.B.; Supervision, D.M.G.; Validation, T.J.B.; Writing —original draft, D.M.G.; Writing—review and editing, O.S. and T.J.B. All authors have read and agreed to the published version of the manuscript.

Funding: This research received no external funding.

Institutional Review Board Statement: Not applicable.

Informed Consent Statement: Not applicable.

Data Availability Statement: The data presented in this study are available in the article.

Acknowledgments: The authors would like to thank S. Anthony and M. Hoffmann for their assistance in the preparation of the samples and measurement of the film thicknesses.

Conflicts of Interest: The authors declare no conflict of interest.

\section{References}

1. Beck, N.; Wyrsch, N.; Hof, C.; Shah, A. Mobility lifetime product-A tool for correlating a-Si:H film properties and solar cell performances. J. Appl. Phys. 1996, 79, 9361-9368. [CrossRef]

2. Asensi, J.; Merten, J.; Voz, C.; Andreu, J. Analysis of the role of mobility-lifetime products in the performance of amorphous silicon p-i-n solar cells. J. Appl. Phys. 1999, 85, 2939-2951. [CrossRef]

3. Wang, H.; Huang, J.; Xing, S.; Yu, J. Improved mobility and lifetime of carrier for highly efficient ternary polymer solar cells based on TIPS-pentacene in PTB7: PC 71 BM. Org. Elect. 2016, 28, 11-19. [CrossRef]

4. Giromali, M.; Bellucci, A.; Calvani, P.; Trucchi, D.M. Large single-crystal diamond substrates for ionizing radiation detection. Phys. Stat. Sol. A 2016, 213, 2634-2640. [CrossRef]

5. Pacansky, J.; Coufal, H.C.; Brown, D.W. The photocyclization of a hydrazone to an indazole. J. Photochem. 1987, 37, $293-313$. [CrossRef]

6. Stasiak, J.W.; Storch, T.J. Hole mobilities in photochemically modified DEH-doped polycarbonate. J. Imaging Sci. Technol. 1996, 40, 299-303.

7. Goldie, D.M. Trap Generation Dynamics in Photo-Oxidised DEH Doped Polymers. Coatings 2015, 5, 263-277. [CrossRef]

8. Goldie, D.M. Cellular Automata Modelling of Photo-Induced Oxidation Processes in Molecularly Doped Polymers. Coatings 2016, 6, 55. [CrossRef]

9. Serad, G.E.; Freeman, B.D.; Stewart, M.E.; Hill, A.J. Gas and vapour sorption and diffusion in poly(ethylene terphthalate). Polymer 2001, 42, 6929-6943. [CrossRef]

10. Hermandez-Rivera, M.A.; Gacia de la Mora, G.; Likhatchev, D.; de la Cruz-Guerra, C.; Diazz, L.L.; Lopez-Castillo, N.N.; Cruz-Gomez, M.J. Oxygen and carbon dioxide transport through high barrier polyester blends. Polym. Eng. Sci. 2009, 49, 1636-1641. [CrossRef]

11. Vyazovkin, V.L.; Korolev, V.V.; Syutkin, V.M.; Tolkatchev, V.A. On oxygen diffusion in poly(methyl methacrylate) films. React. Kinet. Catal. Lett. 2002, 77, 293-299. [CrossRef]

12. Rharbi, Y.; Yekta, A.; Winnik, M.A. A method for measuring oxygen diffusion and oxygen permeation in polymer films based upon fluorescene quenching. Anal. Chem. 1999, 71, 5045-5053. [CrossRef]

13. Pacansky, J.; McLean, A.D.; Miller, M.D. Theoretical Calculation and Experimental Studies on the Electronic Structures of Hydrazones and Hydrazone Radical Cations: Formaldehyde Hydrazone and Benzaldehyde Diphenylhydrazones. J. Phys. Chem. 1990, 94, 90-98. [CrossRef]

14. Goldie, D.M. Organic Charge Transport Materials for Xerographic Imaging. In Handbook of Photochemistry and Photobiology; Nalwa, H.S., Ed.; American Scientific Publishers: Valencia, CA, USA, 2003; Volume 2, pp. 195-256.

15. Pizarro, G.D.C.; Marambio, O.G.; Jeria-Orell, M.; Oyarzún, D.P.; Martin-Trasanco, R.; Sánchez, J. Porous Surface Films with Tunable Morphologies and Hydrophobic Properties Based on Block Copolymer Under the Effects of Thermal Annealing. Front. Chem. 2019, 7, 181. [CrossRef]

16. Cabrera, J.N.; Ruiz, M.M.; Fascio, M.; D’Accorso, N.; Mincheva, R.; Dubois, P.; Lizarraga, L.; Negri, R.M. Increased Surface Roughness in Polydimethylsiloxane Films by Physical and Chemical Methods. Polymers 2017, 9, 331. [CrossRef]

17. Molyneux, P. "Transition-site" model for the permeation of gases and vapors through compact films of polymer. J. Appl. Polym. Sci. 2001, 79, 981-1024. [CrossRef] 